\title{
15-Hydroxyprostaglandin Dehydrogenase Is Associated with the Troglitazone-Induced Promotion of Adipocyte Differentiation in Human Bone Marrow Mesenchymal Stem Cells
}

\author{
Minsoo $\mathrm{NoH}^{1, *}$, and Soo Hwan LEE ${ }^{2}$ \\ ${ }^{1}$ AmorePacific Corporation Research and Development Center, Yongin 446-729, \\ ${ }^{2}$ Department of Physiology, Ajou University School of Medicine, Suwon 443-749, Republic of Korea
}

(Received December 4, 2009; Revised December 21, 2009; Accepted January 7, 2010)

\begin{abstract}
Adipocyte differentiation in human bone marrow mesenchymal stem cells (hBM-MSCs) is not as efficient as that in murine pre-adipocytes when induced by adipogenic agents including insulin, dexamethasone, and 3-isobutyl-1-methylxanthine (IDX condition). Therefore, the promotion of adipocyte differentiation in hBM-MSCs has been used as a cell culture model to evaluate insulin sensitivity for anti-diabetic drugs. In hBM-MSCs, PPAR $\gamma$ agonists or sulfonylurea anti-diabetic drugs have been added to IDX conditions to promote adipocyte differentiation. Here we show that troglitazone, a peroxisome proliferator-activated receptor-gamma (PPAR $\gamma$ ) agonist, significantly reduced the levels of anti-adipogenic $\mathrm{PGE}_{2}$ in IDX-conditioned hBM-MSC culture supernatants when compared to $\mathrm{PGE}_{2}$ levels in the absence of PPAR $\gamma$ agonist. However, there was no difference in the mRNA levels of cyclooxygenases (COXs) and the activities of COXs and prostaglandin synthases during adipocyte differentiation in hBM-MSCs with or without troglitazone. In hBM-MSCs, troglitazone significantly increased the mRNA level of 15-hydroxyprostaglandin dehydrogenase (HPGD) which can act to decrease PGE 2 levels in culture. These results suggest that the role of PPAR $\gamma$ activation in promoting adipocyte differentiation in hBM-MSCs is to reduce anti-adipogenic $\mathrm{PGE}_{2}$ levels through the up-regulation of HPGD expression.
\end{abstract}

Keywords: Human bone marrow mesenchymal stem cells, Adipocyte differentiation, PPAR $\gamma, \mathrm{PGE}_{2}$, Troglitazone, 15-hydroxyprostaglandin dehydrogenase

\section{INTRODUCTION}

Human bone marrow mesenchymal stem cells (hBMMSCs) are widely distributed in various tissues and organs and can differentiate into adipocytes (Pittenger et al., 1999; Janderova et al., 2003). In obesity, it has been implicated that hBM-MSCs are recruited to a new adipocyte pool by metabolic changes in vivo (Entenmann and Hauer, 1996; Sakaguchi et al., 2005). The adipocyte differentiation of hBM-MSCs has been exploited to study insulin sensitivity, obesity, and related metabolic diseases in human (Janderova et al., 2003; Shin et al., 2009a; Shin et al., $2009 \mathrm{~b})$. In murine pre-adipocytes, the addition of insulin, dexamethasone, and 3-isobutyl-1-methyxanthine (IDX con-

${ }^{*}$ Corresponding author

Tel: +82-31-280-5960 Fax: +82-31-899-2595

E-mail: minsoonoh@amorepacific.com dition) is sufficient to convert virtually all cells into fully differentiated adipocytes (Rosen, 2005). In contrast, it has been shown that less than $10 \%$ of hBM-MSCs in culture undergo adipocyte differentiation in response to IDX conditions (Entenmann et al., 1996; Janderova et al., 2003; Shin et al., 2009a). For hBM-MSCs, the activation of PPAR $\gamma$ is essential to significantly promote adipocyte differentiation and increase the number of differentiated cells. In this regard, adipocyte differentiation of hBM-MSCs has been used as a cell culture model to evaluate effects of anti-diabetic drugs like PPAR $\gamma$ agonists and sulfonylurea anti-diabetics on insulin sensitivity (Janderova et al., 2003; Shin et al., 2009a).

hBM-MSCs constitutively express both cyclooxygeanse-1 (COX-1) and COX-2 and produce prostaglandins (PGs) in culture (Arikawa et al., 2004). $\mathrm{PGE}_{2}$ has been known to inhibit adipocyte differentiation (Gregoire et al., 1998; Yan et al., 2003); therefore $\mathrm{PGE}_{2}$ synthesized by 
hBM-MSCs can affect hBM-MSCs in an autocrine or paracrine manner. The transcriptional and translational changes of COXs and $\mathrm{PGE}_{2}$ synthases during adipocyte differentiation have been extensively studied in murine 3T3-L1 and Ob1771 pre-adipocytes (Borglum et al., 1999; Yan et al., 2003; Tsuboi et al., 2004). However, the expression profile of prostanoid enzymes and their roles in adipocyte differentiation are not fully documented in hBM-MSCs.

In the present study, we evaluate $\mathrm{PGE}_{2}$ synthesis, the gene expression pattern of $\mathrm{PGE}_{2}$ synthetic and catabolizing enzymes during adipocyte differentiation in hBM-MSCs to study the effect of PPAR $\gamma$ agonists on the prostanoid pathway. Our study suggests that the role of PPAR $\gamma$ agonists in promoting the adipocyte differentiation of hBMMSCs may be associated with the up-regulation of 15- hydroxyprostaglandin dehydrogenase (HPGD) gene expression.

\section{MATERIALS AND METHODS}

\section{Cell culture and differentiation of hBM-MSCs}

hBM-MSCs were purchased from Lonza, Inc. (Walkersville, MD, USA). hBM-MSCs were maintained and differentiated into adipocytes according to the manufacturer's instructions with minor modifications (Janderova et al., 2003; Shin et al., 2009a; Shin et al., 2009b). Briefly, hBM-MSCs were maintained in Dulbecco- modified eagle's medium (DMEM) with low glucose ( $1 \mathrm{~g} / \mathrm{L}$ glucose) containing $10 \%$ fetal bovine serum (FBS) (Lonza, Walkersville, MD, USA), supplemented with antibiotics and Glutamax $^{\mathrm{TM}}$ (Invitrogen, Carlsbad, CA, USA). hBM-MSCs were plated at a density of 3 to $5 \times 10^{3} / \mathrm{cm}^{2}$ and grown at $37^{\circ} \mathrm{C}$ in a humidified cell culture incubator under $5 \% \mathrm{CO}_{2}$. To induce adipocyte differentiation, hBM-MSCs were grown to $100 \%$ confluence in the growth medium. Three days after the confluence, the growth medium was changed with DMEM with high glucose ( $4.5 \mathrm{~g} / \mathrm{L}$ glucose) supplemented with $10 \%$ FBS, GlutamaxTM, $10 \mu \mathrm{g} / \mathrm{ml}$ insulin, $1 \mu \mathrm{M}$ dexamethasone, $0.5 \mathrm{mM}$ 3-isobutyl-1-methylxanthine (IBMX), and indomethacin/troglitazone. After the induction of adipocyte differentiation, media were changed with the adipogenesis-inducing medium at every 48 hours.

\section{Total RNA isolation and quantitative real-time reverse tra- nscription polymerase chain reaction (Q-RT-PCR)}

Q-RT-PCR was performed using Assays-on-Demand ${ }^{\mathrm{TM}}$ Gene Expression kits (Applied Biosystems, Foster City, CA). cDNA samples were analyzed for COX-1 (Hs00924803_m1), COX-2 (Hs00153133_m1), 15-HPGD (Hs00168359_m1), prostaglandin E synthase 1 (PTGES, Hs- 00610420_m1), prostaglandin E synthase 2 (PTGES2, Hs00228159_m1), prostaglandin E synthase 3 (PTGES3, Hs00832847_gH), fatty acid binding protein 4 (FABP4, Hs00609791_m1), and peroxisome proliferator activated

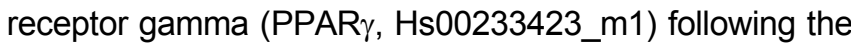
manufacturer's instructions. The housekeeping gene, glyceraldehyde-3-phosphate dehydrogenase (GAPDH, 4333764F), was analyzed (Applied Biosystems) as control. The cDNA from the reverse transcription reaction was amplified by PCR to measure the FAM fluorescence of each PCR cycle using the Corbett Research Rotor-Gene 6 Detection System (Corbett Research, Mortlake, NSW, Australia). Quantification of the relative differences among various adipogenic conditions was calculated using equations from a mathematical model developed by Pfaffl (Pfaffl, 2001).

\section{Measurement of COX activity in culture and $\mathrm{PGE}_{2}$ enzyme immunoassay (EIA)}

After the treatment of adipogenic conditions for 24 hours, media were harvested and centrifuged at 12,000 $\mathrm{rpm}, 4^{\circ} \mathrm{C}$ for 10 minutes, and supernatants were used for the measurement of accumulated levels of $\mathrm{PGE}_{2}$. To measure the activity of COXs in cell culture, hBM-MSCs were washed twice with phosphate buffered saline (PBS) and incubated with $10 \mu \mathrm{M}$ of arachidonic acid in DMEM without serum at $37^{\circ} \mathrm{C}$ for 10 minutes. Enzyme reactions were stopped by addition of $1 \mathrm{~N} \mathrm{NaOH}$ and neutralized with $1 \mathrm{~N} \mathrm{HCl}$, and concentrations of prostaglandins were measured with enzyme immunoassay (EIA) systems from Cayman Chemical Co. (Ann Arbor, MI, USA).

\section{Oil Red O staining}

Cells were washed in PBS, fixed for 30 minutes in 10\% formalin solution in PBS. Fixed cells were washed in $60 \%$ isopropyl alcohol and stained at room temperature with Oil Red O in 60\% isopropyl alcohol. After 10 minute-Oil Red O staining, cells were quickly washed in distilled water. To determine the adipogenic level, adsorbed Oil Red O stain was dissolved with $100 \%$ isopropyl alcohol and absorbance was measured at $500 \mathrm{~nm}$.

\section{Statistical analysis}

All statistical analyses were performed with MINITAB ${ }^{\circledR}$ software (Minitab Inc. State College, PA, USA). Results are expressed as the means \pm standard deviation. Statistical analyses were performed with Student's $t$-test for comparison with control. 


\section{RESULTS}

\section{PPAR $\gamma$ activation decreased the level of anti-adipogenic $\mathrm{PGE}_{2}$ in culture during adipocyte differentiation in $\mathrm{hBM}$ - MSCs}

We examined the level of adipogenic differentiation in hBM-MSCs under various conditions by measuring Oil Red $O$ staining (Fig. 1A). Less than $10 \%$ of IDX-condition treated hBM-MSCs exhibited adipocyte phenotypes after 15 days in adipogenic culture, which was consistent with previous reports (Janderova et al., 2003; Shin et al., 2009a). It was previously shown that the addition of the IDX agents converted nearly all murine pre-adipocytes such as 3T3-L1 cells into adipocytes (Rosen, 2005). In contrast to the adipocyte differentiation of murine pre-adipocytes, the maximum ratio of hBM-MSCs undergoing adipocyte differentiation reached approximately $50 \%$ of hBM-MSCs in culture after 15 days of adipogenic stimulation in the presence of PPAR $\gamma$ agonists, indomethacin (high concentration) and troglitazone. In order to examine whether there was a difference in $\mathrm{PGE}_{2}$ production among various adipogenic conditions, we measured $\mathrm{PGE}_{2}$ levels in culture supernatants accumulated for 24 hours at the first and fifteenth day of adipogenic stimulation. The reduction of $\mathrm{PGE}_{2}$ level in the presence of indomethacin, a strong COX inhibitor, was expected (Fig. 1B). In addition, we found that the $\mathrm{PGE}_{2}$ level was significantly reduced in the presence of troglitazone when compared with that of the IDX condition without a PPAR $\gamma$ agonist (Fig. 1B). Interestingly, the IDX condtion without a PPAR $\gamma$ agonist did not decrease anti-adipogenic $\mathrm{PGE}_{2}$ in $\mathrm{hBM}-\mathrm{MSC}$ culture. Therefore, these data suggest that the supplementation of PPAR $\gamma$ agonists to promote adipocyte differentiation in hBM-MSCs is associated with the decrease in anti-adipogenic $\mathrm{PGE}_{2}$ levels in the IDX condition without a PPAR $\gamma$ agonist.

\section{$\mathrm{PGE}_{2}$ levels in hBM-MSC culture did not correlate with ge- ne expression of either COX-1 or COX-2 during adipocyte differentiation}

It has been reported that gene and protein expression of COXs is down-regulated during adipogenesis in murine 3T3-L1 cells (Yan et al., 2003; Xie et al., 2006). When we examined the expression profile of COX genes during adipocyte differentiation, surprisingly, mRNA levels of both COX-1 and COX-2 were significantly up-regulated upon stimulation of adipocyte differentiation in hBM-MSCs when compared to that of the control (Fig. 2A, B). We also measured the mRNA levels of adipocyte differentiation markers, PPAR $\gamma$ and FABP4, to confirm the differentiation of hBMMSCs (Fig. 2C, D). Interestingly, in spite of the significant
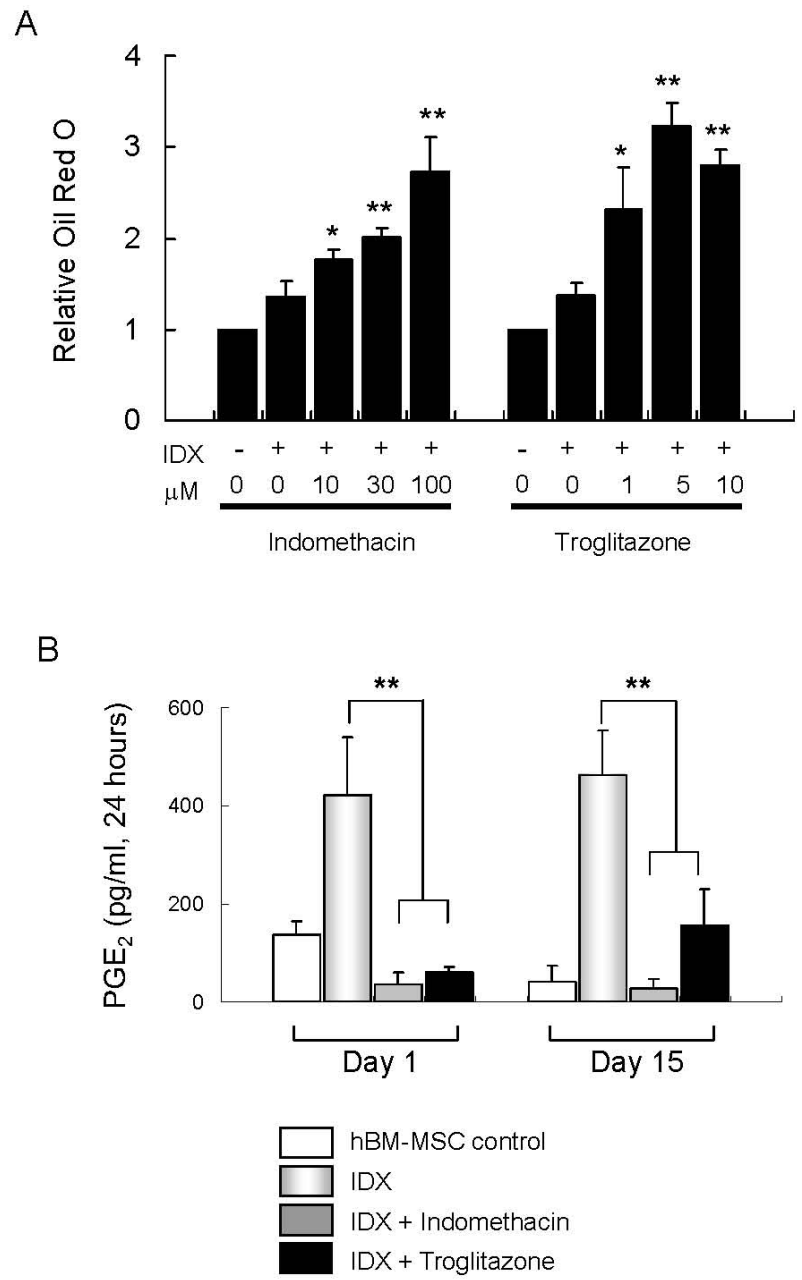

Fig. 1. Adipogenesis in hBM-MSCs. (A) Cells were differentiated in the presence of varying concentrations of indomethacin and troglitazone. Lipid accumulation in hBM-MSCs was determined by Oil Red $O$ staining. Results are presented as the mean \pm standard deviation (SD) of three independent measurements. (B) Concentrations of $\mathrm{PGE}_{2}$ were measured at day 1 and day 15 following adipogenic induction. Results are presented as the mean \pm standard deviation (SD) from quadruplicate measurements. T-tests were also performed to compare the IDX conditions in the absence and presence of PPAR $\gamma$ agonists. *Indicates $p<0.05$, and ${ }^{* *} p<0.01$.

reduction in $\mathrm{PGE}_{2}$ levels (Fig. 1), the level of either COX-1 or COX-2 gene expression was not different among adipogenic IDX conditions regardless of the presence of PPAR $\gamma$ agonists. In other words, troglitazone and indomethacin did not make any difference in COX gene expressions among various adipogenic conditions (Fig. 2). 

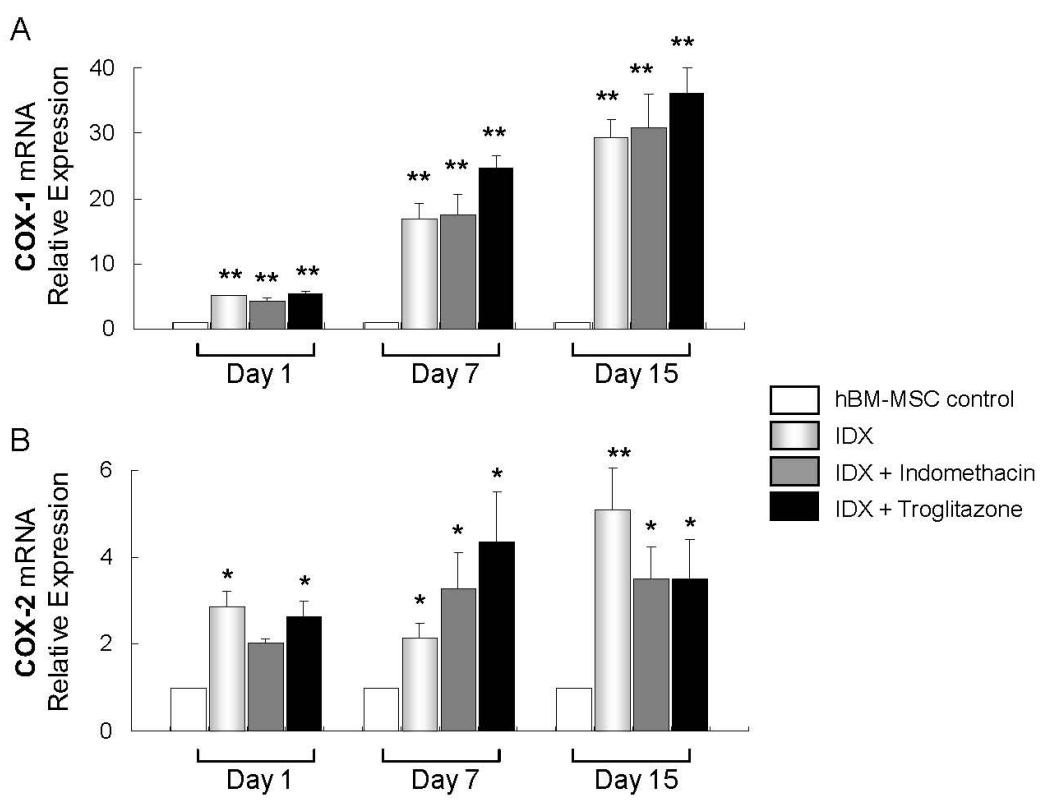

C
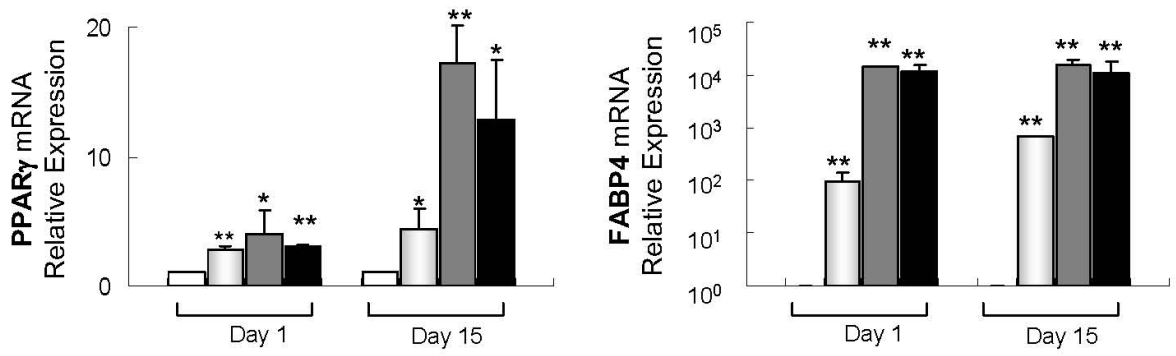

Fig. 2. Up-regulation of COX gene expression during adipogenesis in $\mathrm{hBM}$ MSCs. The mRNA levels of (A) COX-1 and $(B)$ COX-2 were measured by $Q$ RT-PCR at day 1 , day 7 , and day 15 of adipogenic stimulation. The mRNA levels of (C) PPAR $\gamma$ and (D) FABP4 were measured at day 1 and day 15 of adipogenic stimulation. GAPDH was used as an internal control for standardization. Results are presented as the mean $\pm S D(n=4)$. The non-adipogenic condition, insulin treatment only, was used as a control for the Students's $t$-tests. *Indicates $p<0.05$, and ${ }^{* *} p<$ 0.01 .

\section{Down-regulation of prostaglandin E synthase 1 may not sufficiently explain the decrease in $\mathrm{PGE}_{2}$ levels observed during adipocyte differentiation in hBM-MSCs}

In order to understand how $\mathrm{PGE}_{2}$ levels were decreased in spite of an up-regulation of COX-1 and COX-2 mRNAs during adipocyte differentiation, we examined transcriptional changes in $\mathrm{PGE}_{2}$ synthase isoforms (Fig. 3A). There are three prostaglandin $E$ synthase subtypes: prostaglandin E synthase 1 (PTGES1, microsomal prostaglandin E synthase), prostaglandin E synthase 2 (PTGES2), and prostaglandin E synthase 3 (PTGES3, cytosolic prostaglandin E synthase) (Murakami et al., 2000; Tanikawa et al., 2002; Murakami et al., 2003). As shown in Fig. 3, mRNA levels of PTGES1 were significantly decreased in all adipogenic conditions regardless of the presence of PPAR $\gamma$ agonists after day 15 of stimulation (Fig. 3A). In contrast, there was no difference in gene transcription of both PTGES2 and PTGES3 during adipocyte differen- tiation.

Because mRNA levels do not always correlate with corresponding protein function, we measured the enzyme activities of COX and PTGES in cultured hBM-MSCs after addition of arachidonic acid for 10 minutes (Fig. 3B). As mentioned earlier, it was expected that $\mathrm{PGE}_{2}$ synthesis was inhibited in the presence of indomethacin in hBMMSCs (Fig. 3B). In contrast to that of indomethacin, the enzyme activities necessary to synthesize $\mathrm{PGE}_{2}$ were shown to be intact in hBM-MSCs in the presence of troglitazone (Fig. 3B). These data suggest that there may be a specific mechanism by which troglitazone leads to the reduced $\mathrm{PGE}_{2}$ accumulation although it does not directly inhibit COXs or PTGESs during adipogenesis in hBM-MSCs. In this context, it is reasonable to infer the possible involvement of $\mathrm{PGE}_{2}$ synthetic or catabolizing mechanisms in the reduction of $\mathrm{PGE}_{2}$ accumulation in the presence of troglitazone. 
A
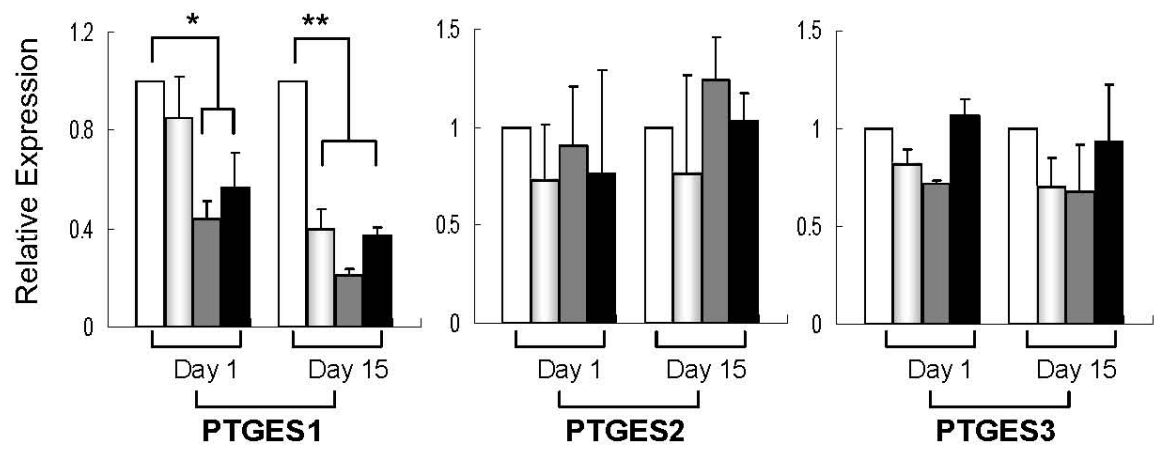

$$
\begin{aligned}
& \text { hBM-MSC control } \\
& \text { IDX } \\
& \text { IDX + Indomethacin } \\
& \text { IDX + Troglitazone }
\end{aligned}
$$

B

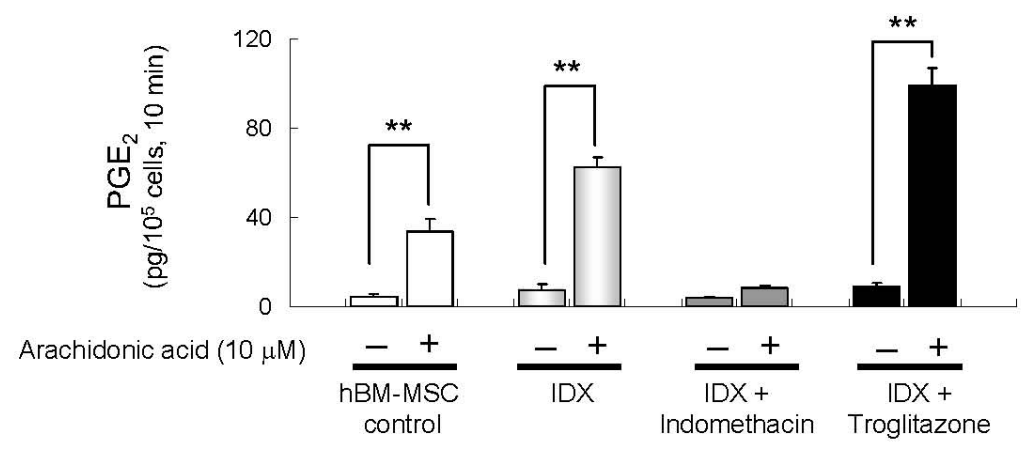

Fig. 3. Relative gene expression levels of $\mathrm{PGE}_{2}$ synthases during adipogenesis in hBM-MSCs. (A) The gene expression level of microsomal $\mathrm{PGE}_{2}$ synthase (PTGES1), PTGES2, and PTGES3, measured by Q-RT-PCR. Results are presented as the mean \pm $\mathrm{SD}(\mathrm{n}=4)$. (B) Cellular $\mathrm{PGE}_{2}$ synthesis, indicative of COX and PTGES enzyme activities, was measured in hBM-MSCs. After day 15 of adipogenic stimulation, cells were washed with PBS and incubated with $10 \mu \mathrm{M}$ arachidonic acid (AA) for 10 minutes. *Indicates $p<0.05$, and ${ }^{* *} p<0.01$.

\section{Up-regulation of $\mathrm{PGE}_{2}$ catabolic enzyme 15-hydroxypro- staglandin dehydrogenase may be pivotal for the decrea- se of $\mathrm{PGE}_{2}$ levels during adipocyte differentiation in $\mathrm{hBM}$ - MSCs}

In order to elucidate the cellular mechanism for the troglitazone-induced down-regulation of $\mathrm{PGE}_{2}$ during adipocyte differentiation in hBM-MSCs, we evaluated the gene expression level of one of $\mathrm{PGE}_{2}$ metabolizing enzymes, 15-hydroxyprostaglandin dehydrogenase (HPGD). HPGD, a $\mathrm{PGE}_{2}$ catabolic enzyme, converts the 15-hydroxyl group of prostaglandins into a keto group (Braithwaite and Jarabak, 1975). When gene expression was measured by Q-RT-PCR, HPGD mRNA levels were shown to be significantly up-regulated in the presence of indomethacin and troglitazone in the IDX condition compared with that in the control (Fig. 4). Most importantly, the HPGD mRNA level in the IDX condition in the absence of PPAR $\gamma$ agonists was unchanged compared with that of the control hBM-MSC culture. Therefore, the induction of HPGD gene transcription by PPAR $\gamma$ agonists in hBM-MSCs may be one of most significant factors to promote the down-regulation of anti-adipogenic $\mathrm{PGE}_{2}$ levels in the adipogenic culture condition.

\section{DISCUSSION}

Prostaglandins play roles as autocrines and/or paracrines to regulate adipocyte differentiation and its function (Gregoire et al., 1998; Reginato et al., 1998; Xie et al., 2006). In this study, we evaluated the $\mathrm{PGE}_{2}$ levels in hBM-MSCs during adipocyte differentiation and analyzed the mRNA expressions of COXs, $\mathrm{PGE}_{2}$ synthases, and HPGD. We found that the requirement of PPAR $\gamma$ activation for promoting adipocyte differentiation in hBM-MSCs may be associated with PPAR $\gamma$-dependent up-regulation of the HPGD gene expression. In murine pre-adipocytes, adipogenic differentiation was followed by a significant reduction in $\mathrm{PGE}_{2}$ level (Yan et al., 2003; Tsuboi et al., 2004; Xie et 


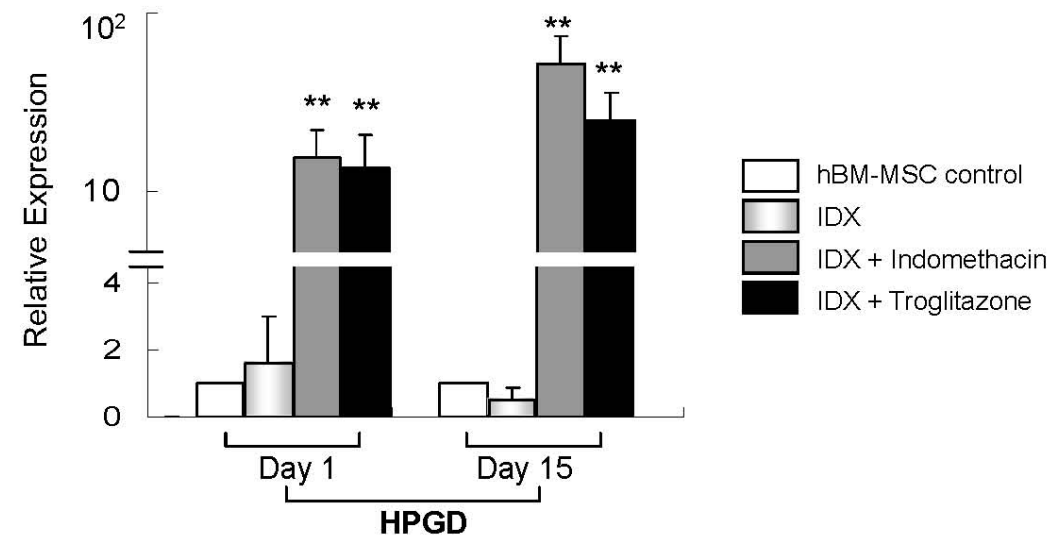

Fig. 4. The role of HPGD gene expression during adipogenesis in hBM-MSCs. HPGD gene expression was significantly increased in adipogenic conditions in the presence of PPAR $\gamma$ agonists. The graph below the double break is represented in arithmetic scale, and above the double break in logarithmic scale. Results are presented as the mean $\pm \mathrm{SD}(\mathrm{n}=3)$. GAPDH was used as an internal control for standardization. The non-adipogenic condition, insulin treatment only, was used as a control for Students's $t$-tests. ${ }^{* *} p<0.01$.

al., 2006). It was reported that $\mathrm{PGE}_{2}$ mediated the suppression of murine 3T3-L1 adipogenesis through prostanoid EP4 receptor signaling (Tsuboi et al., 2004). In contrast, we observed that the $\mathrm{PGE}_{2}$ level in the IDX medium without PPAR $\gamma$ agonist was significantly higher than that of the control hBM-MSC culture (Fig. 1). The PGE 2 level was significantly lower in the presence of troglitazone when compared to the level in the absence of a PPAR $\gamma$ agonist. When exogenously added to hBM-MSC cultures, low nanomolar concentrations of $\mathrm{PGE}_{2}$ inhibited adipocyte differentiation (data not shown), as reported in those of murine pre-adipocytes (Reginato et al., 1998; Xie et al., 2006). Because hBM-MSCs constitutively express COX-1 and COX-2 (Arikawa et al., 2004), $\mathrm{PGE}_{2}$ generated from hBM-MSCs may function as an intrinsic negative feedback regulator for the differentiation of hBM-MSCs. As shown in Fig. 1, the significant change in anti-adipogenic $\mathrm{PGE}_{2}$ levels induced by troglitazone may explain the characteristic inefficient adipocyte differentiation observed in hBMMSCs under IDX conditions (Fig. 1). These data suggested that indomethacin and troglitazone promote adipocyte differentiation in hBM-MSCs partly through the reduction of $\mathrm{PGE}_{2}$ levels in hBM-MSC culture.

In the simultaneous measurement of COX gene transcription, we found that there was no difference in the mRNA levels of COX-1 and COX-2 during adipocyte differentiation in hBM-MSCs although there was the significant difference in $\mathrm{PGE}_{2}$ levels in the existence of a PPAR $\gamma$ agonist (Fig. 2). The COX mRNA levels during adipocyte differentiation in hBM-MSCs were even up-regulated than that of the hBM-MSC control in our study. In contrast, gene transcriptions of COX-1 and COX-2 during adipocyte differentiation in murine pre-adipocyte systems have been reported unchanged or down-regulated (Yan et al., 2003; Xie et al., 2006). In this regard, COX gene expression pat- terns represent one of the major differences observed during adipocyte differentiation between hBM-MSCs and murine pre-adipocytes. PPAR $\gamma$ agonists have been known to either increase or decrease COX gene expression in a cell type-specific manner (He et al., 1996; Inoue et al., 2000; Yang and Frucht, 2001). For instance, anti-diabetic thiazolidinediones (TZDs) induced COX-2 gene expression in human epidermal keratinocytes, whereas they inhibited COX-2 gene expression in human epithelial cell lines ( $\mathrm{He}$ et al., 1996; Inoue et al., 2000).

PTGES1 transcription was previously shown to be induced by pro-inflammatory stimuli, and it is generally thought that alterations in PTGES1 expression are closely correlated with $\mathrm{PGE}_{2}$ production in the inflammatory milieu (Murakami et al., 2003). Thus, we speculated that the down-regulation of PTGES1 gene expression observed might contribute to the decrease in $\mathrm{PGE}_{2}$ levels during adipocyte differentiation in hBM-MSCs. However, our data show that changes in PTGES1 expression did not coincide with $\mathrm{PGE}_{2}$ production under our experimental conditions, especially in the group treated with IDX condition without troglitazone (Fig. 3A). In contrast to PTGES1, the mRNA expressions of both PTGES2 and PTGES3 were unchanged during adipocyte differentiation in hBM-MSCs (Fig. 3A). Both PTGES2 and PTGES3 are constitutively expressed in various cell types, and utilize $\mathrm{PGH}_{2}$ produced by both COX-1 and COX-2 as a substrate to produce $\mathrm{PGE}_{2}$ (Murakami et al., 2000; Tanikawa et al., 2002; Murakami et al., 2003). Therefore, down-regulation of PTGES1 gene expression may not be a main reason for the decrease in $\mathrm{PGE}_{2}$ levels during adipocyte differentiation.

It was reported that several PPAR $\gamma$ agonists decreased $\mathrm{PGE}_{2}$ in non-small-cell lung cancer cells by increasing the activity of HPGD, although the mechanism for HPGD up-regulation was independent of PPAR $\gamma$ trans-activation 
(Hazra et al., 2007). A recent study showed that 15-keto$\mathrm{PGE}_{2}$, a product of the HPGD enzyme, trans-activated PPAR $\gamma$ and enhanced adipocyte differentiation in 3T3-L1 cells (Chou et al., 2007). In addition, the overexpression of HPGD in 3T3-L1 cells also increased PPAR $\gamma$-responses in a $\mathrm{PGE}_{2}$-dependent manner (Chou et al., 2007). Taken together, these results suggest that the up-regulation of HPGD gene expression by troglitazone plays a role in promoting adipocyte differentiation in hBM-MSCs by catabolizing $\mathrm{PGE}_{2}$ to 15-keto-PGE 2 . The promotion of adipocyte differentiation in hBM-MSCs is related to the improvement of insulin sensitivity and has been used as a cell based model for evaluating anti-diabetic drugs (Janderova et al., 2003; Shin et al., 2009a), it will be interesting to study the role of HPGD in the progression of diabetes and related metabolic diseases in future.

In conclusion, our study suggests that the PPAR $\gamma$ induced promotion of adipocyte differentiation in hBM-MSCs may be partly associated with the HPGD related $\mathrm{PGE}_{2}$ down-regulation, implicating the role of anti-diabetic drugs to regulate the HPGD gene expression in insulin sensitivity and related metabolic diseases.

\section{ACKNOWLEDGMENTS}

We thank Dr. Dong Wook Shin for critical reading of the manuscript.

\section{REFERENCES}

Arikawa, T., Omura, K. and Morita, I. (2004). Regulation of bone morphogenetic protein-2 expression by endogenous prostaglandin $E_{2}$ in human mesenchymal stem cells. J. Cell Physiol. 200, 400-406.

Borglum, J. D., Pedersen, S. B., Ailhaud, G., Negrel, R. and Richelsen, B. (1999). Differential expression of prostaglandin receptor mRNAs during adipose cell differentiation. Prostaglandins and other Lipid Mediat. 57, 305-317.

Braithwaite, S. S. and Jarabak, J. (1975). Studies on a 15hydroxyprostaglandin dehydrogenase from human placenta. J. Biol. Chem. 250, 2315-2318.

Chou, W. L., Chuang, L. M., Chou, C. C., Wang, A. H., Lawson, J. A., FitzGerald, G. A. and Chang, Z. F. (2007). Identification of a novel prostaglandin reductase reveals the involvement of prostaglandin $E_{2}$ catabolism in regulation of peroxisome proliferator-activated receptor gamma activation. J. Biol. Chem. 282, 18162-18172.

Entenmann, G. and Hauer, H. (1996). Relationship between replication and differentiation in cultured human adipocyte precursor cells. Am. J. Physiol. 270, C1011-C1016.

Gregoire, F. M., Smas, C. M. and Sul, H. S. (1998). Understanding adipocyte differentiation. Physiol. Rev. 78, 783809.

Hazra, S., Batra, R. K., Tai, H. H., Sharma, S., Cui, X. and
Dubinett, S. M. (2007). Pioglitazone and rosiglitazone decreases prostaglandin $E_{2}$ in non-small-cell lung cancer cells by up-regulating 15-hydroxysprostaglandin dehydrogenase. Mol. Pharmacol. 71, 1715-1720.

He, G., Sung, Y. M. and Fischer, S. M. (1996). Troglitazone induction of COX-2 expression is dependent on ERK activation in keratinocytes. Prostaglandins Leukotrienes Essent. Fatty Acids 74, 193-197.

Inoue, H., Tanabe, T. and Umesono, K. (2000). Feedback control of cyclooxygenase-2 expression through PPARgamma. J. Biol. Chem. 275, 28028-28032.

Janderova, L., McNeil, M., Murrell, A. N., Mynatt, R. L. and Smith, S. R. (2003). Human mesenchymal stem cells as an in vitro model for human adipogenesis. Obesity Res. 11, 65-74.

Murakami, M., Nakashima, K., Kamei, D., Masuda, S., Ishikawa, Y., Ishii, T., Ohmiya, Y., Watanabe, K. and Kudo, I. (2003). Cellular prostaglandin $E_{2}$ production by membrane-bound prostaglandin $\mathrm{E}$ synthase-2 via both cyclooxygenases-1 and -2. J. Biol. Chem. 278, 37937-37947.

Murakami, M., Naraba, H., Tanioka, T., Semmyo, N., Nakatani, Y., Kojima, F., Ikeda, T., Fueki, M., Ueno, A., Ohishi, S. and Kudo, I. (2000). Regulation of Prostaglandin $E_{2}$ Biosynthesis by Inducible Membrane-associated Prostaglandin E2 Synthase That Acts in Concert with Cyclooxygenase-2. J. Biol. Chem. 275, 32783-32792.

Pfaffl. M. W. (2001). A new mathematical model for relative quantification in real-time RT-PCR. Nucleic Acids Res. 29, e45.

Pittenger, M. F., Mackay, A. M., Beck, S. C., Jaiswal, R. K., Douglas, R., Mosca, J. D., Moorman, M. A., Simonetti, D. W., Craig, S. and Marshak, D. R. (1999). Multilineage potential of adult human mesenchymal stem cells. Science 284, 143147.

Reginato, M. J., Krakow, S. L., Bailey, S. T. and Lazar, M. A. (1998). Prostaglandins promote and block adipogenesis through opposing effects on peroxisome proliferator-activated receptor gamma. J. Biol. Chem. 273, 1855-1858.

Rosen, E. D. (2005). The transcriptional basis of adipocyte development. Prostaglandins Leukotrienes Essent. Fatty Acids 73, 31-34.

Sakaguchi, Y., Sekiya, I., Yagishita, K. and Takeshi, M. (2005). Comparison of human stem cells derived from various mesenchymal tissues. Arthritis Rheum. 52, 2521-2529.

Shin, D. W., Kim, S. N., Lee, S. M., Lee, W., Song, M. J., Park, S. M., Lee, T. R., Baik, J. H., Kim, H. K., Hong, J. H. and Noh, M. (2009a). (-)-Catechin promotes adipocyte differentiation in human bone marrow mesenchymal stem cells through PPARgamma transactivation. Biochem. Pharmacol. 77, 125133.

Shin, J. H., Shin, D. W. and Noh, M. (2009b). IL-17A inhibits adipocyte differentiation in human mesenchymal stem cells and regulates pro-inflammatory responses in adipocytes. Biochem. Pharmacol. 77, 1835-1844.

Tanikawa, N., Ohmiya, Y., Ohkubo, H., Hashimoto, K., Kangawa, K., Kojima, M., Ito, S. and Watanabe, K. (2002). Identification and characterization of a novel type of membrane-associated prostaglandin E synthase. Biochem. Biophys. Res. Comm. 291, 884-889.

Tsuboi, H., Sugimoto, Y., Kainoh, T. and Ichikawa, A. (2004). Prostanoid EP4 receptor is involved in suppression of 3T3- 
L1 adipocyte differentiation. Biochem. Biophys. Res. Comm. 322, 1066-1072.

Xie, Y., Kang, X., Ackerman, W. E., Belury, M. A., Koster, C., Rovin, B. H., Landon, M. B. and Kniss, D. A. (2006). Differentiation-dependent regulation of the cyclooxygenase cascade during adipogenesis suggests a complex role for prostaglandins. Diabetes Obesity Metab. 8, 83-93.
Yan, H., Kermouni, A., Abdel-Hafez, M. and Lau, D. C. W. (2003). Role of cyclooxygenases COX-1 and COX-2 in modulating adipogenesis in 3T3-L1 cells. J. Lipid Res. 44, 424-429.

Yang, W. L. and Frucht, H. (2001). Activation of the PPAR pathway induces apoptosis and COX-2 inhibition in HT-29 human colon cancer cells. Carcinogenesis 22, 1379-1383. 